\title{
Management of amphotericin-induced phlebitis among HIV patients with cryptococcal meningitis in a resource- limited setting: a prospective cohort study
}

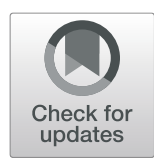

Cynthia Ahimbisibwe ${ }^{1}$, Richard Kwizera ${ }^{1 *}$ (D), Jane Frances Ndyetukira ${ }^{1}$, Florence Kugonza ${ }^{1}$, Alisat Sadiq ${ }^{1}$, Kathy Huppler Hullsiek2, Darlisha A. Williams ${ }^{1,3}$, Joshua Rhein ${ }^{1,3}$, David R. Boulware ${ }^{3}$ and David B. Meya 1,3,4

\begin{abstract}
Background: Amphotericin-induced phlebitis is a common infusion-related reaction in patients managed for cryptococcal meningitis. High-quality nursing care is critical component to successful cryptococcosis treatment. We highlight the magnitude and main approaches in the management of amphotericin-induced phlebitis and the challenges faced in resource-limited settings.

Methods: We prospectively determined the incidence of amphotericin-induced phlebitis during clinical trials in Kampala, Uganda from 2013 to 2018. We relate practical strategies and challenges faced in clinical management of phlebitis.

Results: Overall, 696 participants were diagnosed with HIV-related cryptococcal meningitis. Participants received 714 doses of intravenous (IV) amphotericin B deoxycholate $0.7-1.0 \mathrm{mg} / \mathrm{kg} /$ day for induction therapy through peripheral IV lines at a concentration of $0.1 \mathrm{mg} / \mathrm{mL}$ in 5\% dextrose. Overall, 18\% (125/696) developed amphotericininduced phlebitis. We used four strategies to minimize/prevent the occurrence of phlebitis. First, after every dose of amphotericin, we gave one liter of intravenous normal saline. Second, we rotated IV catheters every three days. Third, we infused IV amphotericin over $4 \mathrm{~h}$. Finally, early ambulation was encouraged to minimize phlebitis. To alleviate phlebitis symptoms, warm compresses were used. In severe cases, treatment included topical diclofenac gel and oral anti-inflammatory medicines. Antibiotics were used only when definite signs of infection developed. Patient/caregivers' education was vital in implementing these management strategies. Major challenges included implementing these interventions in participants with altered mental status and limited access to topical and oral anti-inflammatory medicines in resource-limited settings.
\end{abstract}

Conclusions: Amphotericin-induced phlebitis is common with amphotericin, yet phlebitis is a preventable complication even in resource-limited settings.

Trial registration: The ASTRO-CM trial was registered prospectively. ClincalTrials.gov: NCT01802385; Registration date: March 1, 2013; Last verified: February 14, 2018.

Keywords: Phlebitis, Thrombophlebitis, Amphotericin B, Cryptococcal infection, HIV, Nursing, Sub-Saharan Africa

\footnotetext{
*Correspondence: kwizerarichard@ymail.com

${ }^{1}$ Infectious Diseases Institute, College of Health Sciences, Makerere University,

Kampala, Uganda

Full list of author information is available at the end of the article
}

(c) The Author(s). 2019 Open Access This article is distributed under the terms of the Creative Commons Attribution 4.0 International License (http://creativecommons.org/licenses/by/4.0/), which permits unrestricted use, distribution, and reproduction in any medium, provided you give appropriate credit to the original author(s) and the source, provide a link to the Creative Commons license, and indicate if changes were made. The Creative Commons Public Domain Dedication waiver (http://creativecommons.org/publicdomain/zero/1.0/) applies to the data made available in this article, unless otherwise stated. 


\section{Background}

Cryptococcal meningitis accounts for 15 to $25 \%$ of AIDS-related deaths [1-3]. Treatment of cryptococcal meningitis currently relies on amphotericin $\mathrm{B}$ based regimens. The 2018 World Health Organization (WHO) guidelines recommend a one-week induction regimen with amphotericin B deoxycholate and flucytosine for treating cryptococcal meningitis among human immunodeficiency virus (HIV) patients [4]. However, in the absence of flucytosine, 2 weeks of amphotericin B deoxycholate and high dose fluconazole is recommended. This is followed by a consolidation and maintenance phase using oral fluconazole monotherapy.

In high-resource settings, intravenous (IV) administration of amphotericin B deoxycholate is typically given through a central line. However, in resource-limited settings, amphotericin is typically administered via peripheral venous access due to limited supplies. Amphotericin has toxicity with severe side effects [5]. These infusion-related side effects include nephrotoxicity, nausea, vomiting, fevers, chills, hypotension and phlebitis [6]. Phlebitis is a common, potentially severe infusion-related reaction. Phlebitis may be directly associated with infusion of amphotericin, as well as other intravenous medications commonly used to prevent amphotericin-induced toxicity, such as potassium chloride. Phlebitis involves inflammation of the veins due to damage to the blood vessel walls, coagulation abnormality, or impaired venous blood flow. Phlebitis is characterized by pain, redness, tenderness and swelling of the affected area at the infusion site. In severe cases, infusion-associated phlebitis can lead to painful discomfort for the patient, undesirable interruption of the course of amphotericin treatment, possible thrombosis, or sepsis. All of these could negatively affect treatment outcomes.

High-quality nursing care is a critical component when administering amphotericin B for many AIDS-related opportunistic infections, including cryptococcosis, histoplasmosis, or talaromycosis [7]. Herein, we prospectively determined the incidence of amphotericin-induced phlebitis and described our practical experiences and challenges in managing it in a resource-limited setting during a prospective multi-site randomized clinical trial managing participants co-infected with HIV and cryptococcal meningitis.

\section{Methods}

The current study reported in this manuscript was an observational cohort study that was nested under the Adjunctive Sertraline for the Treatment of HIVassociated Cryptococcal Meningitis (ASTRO-CM) Clinical Trial (ClincalTrials.gov: NCT01802385) [8]. Therefore, we adhered to the STROBE guidelines. Eligibility for this observational study was the same as that for the ASTRO-CM trial. We enrolled a prospective cohort of subjects screened for the ASTRO-CM Clinical Trial. This encompassed a phase II trial followed by a phase III randomized clinical trial conducted in Uganda at the Mulago National Referral and Mbarara Hospitals from Sept 2013 to May 2017 [8]. As part of this trial, all persons presenting with suspected meningitis were approached for consent for a diagnostic lumbar puncture. Participants included in the study were HIV-infected, $\geq 18$ years, with a positive CSF CrAg.

Participants were diagnosed with cryptococcal meningitis based on cryptococcal antigen lateral flow antigen (Immy, Norman, Oklahoma) and confirmed with quantitative cerebrospinal fluid (CSF) culture [9, 10]. During the induction phase, patients with confirmed cryptococcal meningitis were treated primarily with amphotericin B deoxycholate $0.7-1.0 \mathrm{mg} / \mathrm{kg} /$ day via peripheral intravenous (IV) access at a concentration of $0.1 \mathrm{mg} / \mathrm{mL}$ in 5\% dextrose. Amphotericin $50 \mathrm{mg}$ was prepared in $500 \mathrm{~mL}$. When participants weighed $<50 \mathrm{~kg}$, volume was wasted before infusion to achieve $1.0 \mathrm{mg} / \mathrm{kg}$ dose. Participants received fluconazole $800-1200 \mathrm{mg}$ per day and other concomitant medications aimed at mitigating the toxic effects of amphotericin (potassium chloride, magnesium chloride, multivitamins, analgesics, and ondansetron anti-emetic) per standardize treatment protocols.

Each participant received 7-14 doses of amphotericin. One liter of saline was administered intravenously immediately prior to amphotericin administration to minimize renal toxicity, and a second 1 liter was administered postinfusion to minimize phlebitis. During this cohort, $\mathrm{KCl}$ tablets $(16 \mathrm{mEq})$ were given to all participants daily up to the end of the induction phase. However, for those that developed severe hypokalemia, we added IV $\mathrm{KCl}(40 \mathrm{mEq})$ in $\mathrm{NaCl} 0.9 \%$ (saline). Less than ten patients (out of 696) used the IV $\mathrm{KCl}$. Clinicians and nurses reviewed participants daily to assess phlebitis, toxicity and patients' clinical progress. Clinical reviews included taking vital signs, general examination, drug adherence, adverse events, reviewing/requesting laboratory tests, and recording any new complaints. In this study, we aimed to determine the incidence of amphotericin-induced phlebitis and describe our practical experiences and challenges in managing it in a resource-limited setting using these enrolled patients.

\section{Ethical consideration}

All research participants or their surrogates provided written informed consent. Ethical approval was obtained from the Uganda National Council of Science and Technology (UNCST), Mulago Hospital Research and Ethics Committee, and the University of Minnesota.

\section{Statistical analysis}

Data were analysed using STATA version 14 (STATA, College Station, Texas). We performed statistical analysis 
to determine the frequency of phlebitis and the distribution of baseline demographic characteristics of the participants. Summaries were made in frequency \& percentages for each baseline characteristic considered as a categorical, and medians (interquartile range) when each characteristic is considered as a continuous variable.

\section{Results}

\section{Summary of participants}

During the ASTRO-CM study, 1739 participants were screened and 696 HIV-infected patients with cryptococcal meningitis were enrolled, of which $40 \%$ were women. The median age for all participants was 35 years (IQR; 30-41). The median CD4 cell count for all participants was 16 cells/ $\mu \mathrm{L}$ (IQR; 6-49). The duration of hospitalization was a median of 14 days (IQR; 9-16). Over the duration of the study, 607 participants presented with an index episode of cryptococcal meningitis while 89 participants presented as second episodes (Table 1).

\section{Incidence of phlebitis}

Overall, $18 \% \quad(125 / 696)$ of participants developed amphotericin-induced phlebitis during hospitalization. Among the patients presenting with an index episode of cryptococcal meningitis, 19\% (118/607) developed phlebitis while only $8 \%(7 / 89)$ of those with second episode meningitis developed phlebitis $(P<0.01)$. In our cohort, the time to development of phlebitis varied widely with
$27 \%$ of the participants developing it in the first week of therapy while majority (43\%) developed it in the second week of therapy. However, $30 \%$ of the participants developed phlebitis after the 2 weeks induction phase (Table 2).

Among demographic factors, the only difference was that baseline CSF culture sterility was associated with less phlebitis $(\mathrm{P}<0.01)$ (Table 1$)$. As part of standardized care, participants with sterile CSF culture only received 7 days of amphotericin thus were less at risk of phlebitis. Only $6 \%(8 / 125)$ of the participants with phlebitis and $4 \%$ (23/571) without phlebitis were given antibiotics during induction due to suspected presence of an infected thrombus. However, even with the increased awareness and attention we gave to phlebitis, its incidence did not reduce over time and the distribution by year was as follows; $21 \%$ in 2013 (26 cases), 13\% in 2014 (16 cases), 8\% in 2015 (10 cases), 26\% in 2016 (33 cases) and 32\% in 2017 (40 cases). This increased rate in 2016-2017 may reflect an observation bias of increased awareness and attention to phlebitis.

\section{Discussion}

\section{Post amphotericin saline infusion}

One liter or more of saline was administered intravenously immediately after amphotericin administration to minimize phlebitis and renal toxicity. Similarly, WHO recommends administration of "one liter of normal saline solution with $20 \mathrm{mEq}$ of potassium chloride $(\mathrm{KCl})$

Table 1 Demographics of cryptococcal meningitis research participants

\begin{tabular}{|c|c|c|c|c|c|c|c|}
\hline \multirow[t]{2}{*}{ Demographics } & \multicolumn{2}{|c|}{ Overall } & \multicolumn{2}{|c|}{ Phlebitis During Induction } & \multicolumn{2}{|c|}{ No Phlebitis During Induction } & \multirow{2}{*}{$\begin{array}{l}P \text { - } \\
\text { value }\end{array}$} \\
\hline & $\mathrm{N}$ & Median [IQR] or N (\%) & $\mathrm{N}$ & Median [IQR] or N (\%) & $\mathrm{N}$ & Median [IQR] or N (\%) & \\
\hline Age, years & 696 & $35[30,41]$ & 125 & $35[30,40]$ & 571 & $35[30,41]$ & 0.53 \\
\hline Women & 696 & $275(39.5 \%)$ & 125 & $47(38 \%)$ & 571 & $228(40 \%)$ & 0.63 \\
\hline Weight, kg & 573 & $52[48,60]$ & 97 & $53[49,60]$ & 476 & $52[48,60]$ & 0.82 \\
\hline Receiving HIV therapy & 695 & $367(53 \%)$ & 125 & 59 (47\%) & 570 & $308(54 \%)$ & 0.17 \\
\hline Glasgow Coma Score $<15$ & 695 & $295(42 \%)$ & 125 & 47 (38\%) & 570 & $248(44 \%)$ & 0.23 \\
\hline Duration of HIV, months & 656 & $4.3[0.3,38.1]$ & 116 & $4.1[0.3,50.8]$ & 540 & $4.3[0.3,37.2]$ & 0.79 \\
\hline CD4 T cell count, cells/ $\mu \mathrm{L}$ & 661 & $16[6,49]$ & 122 & $15[6,50]$ & 539 & $17[6,49]$ & 0.63 \\
\hline Initial Meningitis episode & 696 & $607(87 \%)$ & 125 & $118(94 \%)$ & 571 & $489(86 \%)$ & $<0.01$ \\
\hline Second Meningitis episode & 696 & $89(13 \%)$ & 125 & $7(5.6 \%)$ & 571 & $82(14 \%)$ & \\
\hline CSF Culture, $\log _{10} \mathrm{CFU} / \mathrm{mL}$ & 692 & $4.6[2.8,5.4]$ & 124 & $4.5[3.1,5.3]$ & 568 & $4.6[2.8,5.4]$ & 0.89 \\
\hline Sterile cryptococcal culture & 692 & $74(11 \%)$ & 124 & $5(4.0 \%)$ & 568 & $69(12 \%)$ & $<0.01$ \\
\hline CSF opening pressure, $\mathrm{cm} \mathrm{H}_{2} \mathrm{O}$ & 607 & $27[18,40]$ & 105 & $27[19,38]$ & 502 & $27[18,41]$ & 0.63 \\
\hline Opening pressure $>25 \mathrm{~cm} \mathrm{H}_{2} \mathrm{O}$ & 607 & $336(55 \%)$ & 105 & $55(52 \%)$ & 502 & $281(56 . \%)$ & 0.50 \\
\hline CSF white cells/ $\mu \mathrm{L}$ & 671 & $<5[<5,45]$ & 121 & $<5[<5,45]$ & 550 & $<5[<5,45]$ & 0.12 \\
\hline CSF white cells $\geq 5$ cells $/ \mu \mathrm{L}$ & 671 & $243(36 \%)$ & 121 & $36(30 \%)$ & 550 & $207(38 \%)$ & 0.10 \\
\hline CSF protein, mg/dL & 594 & $49[23,100]$ & 118 & $42[25,100]$ & 476 & $50[22,100]$ & 0.83 \\
\hline
\end{tabular}

Data presented are $\mathrm{N}$ (\%) or medians with interquartile ranges (IQR). Abbreviations: CSF cerebrospinal fluid, CFU colony forming units of Cryptococcus, $\mathrm{N}$ number of participants with data for each parameter 
Table 2 Duration of amphotericin B therapy and timing of Phlebitis during Cryptococcal meningitis

\begin{tabular}{lll}
\hline Characteristic & Phlebitis & No Phlebitis \\
\hline $\mathrm{N}$ & 125 & 571 \\
Days of amphotericin B & $14[12,14]$ & $8[4,12]$ \\
Days in hospital & $15[14,18]$ & $14[8,16]$ \\
Time to phlebitis & & \\
$1-7$ days & $34(27 \%)$ & \\
$8-10$ days & $23(18 \%)$ & \\
$11-14$ days & $31(25 \%)$ & \\
$15-18$ days & $22(18 \%)$ & $23(4.0 \%)$ \\
$>18$ days & $15(12 \%)$ & $9[6,11]$ \\
Received antibiotic for phlebitis & $8(6.4 \%)$ & \\
Time to antibiotic initiation, days & $13[12,15]$ & \\
\hline
\end{tabular}

Limited to amoxicillin, cloxacillin, and doxycycline

over two hours before each controlled infusion of amphotericin $\mathrm{B}$ and one to two $8-\mathrm{mEq} \mathrm{KCl}$ tablets orally twice daily" [4]. The supplemental $\mathrm{KCl}$ is used to prevent hypokalemia and renal insufficiency resulting of potassium wasting. This strategy of giving saline also helped in managing dehydration in this population.

\section{Rotation of the IV infusion site}

Each amphotericin infusion was regulated to run for at least 4 hours at a rate of approximately $120 \mathrm{~mL} / \mathrm{hr}$. $(40$ drops/ minute). However, in our setting, we do not use a standard infusion pump as would be in a higherresourced environment. We use the regulator that is fixed on the IV tubing to help standardize the drip rate. The infusion should not be given too quickly given risk for both rigors and phlebitis with too rapid of an infusion. Previous studies have shown that Infusion concentrations of more than $0.1 \mathrm{~g} / \mathrm{L}$ are associated with increased risks of amphotericin-induced phlebitis; which may be minimized by using a central line [11].

Time to development of amphotericin-induced phlebitis and the factors that affect this time remain unclear. This is because some patients developed the inflammation after one dose while others developed it after additional doses. Previous studies showed that IV antibiotics, female sex, prolonged $(>48 \mathrm{~h}$ ) catheterization and catheter material strongly predicted phlebitis [12]. Another recent study indicated that the incidence of phlebitis was associated with the length of time the catheter remained in place and puncture in the forearm [13].

The IV infusion site was changed after every 3 days or when patients complained of severe pain at the site of IV access or limb swelling, which suggested phlebitis. In most cases, the IV infusion was moved to the other arm, which would give time for the swelling on the arm previously used to resolve. However, in a few cases, both arms would have prolonged phlebitis that required us to move the IV line to the lower limbs to prevent treatment interruption. The main challenge to this method was that patients were usually dehydrated, making it difficult to find a vein to place a cannula for IV infusion. There is also limited ability and capacity to perform venous cutdowns or place central venous catheters. So none of the patients got a central venous catheter. In addition, some patients were disoriented and aggressive. This made it difficult to obtain intravenous access or sustain access. Finally, shortage of infusion sets to allow intravenous access rotation every 3 days is a challenge outside of the research setting in many resource-limited settings.

\section{Ambulation}

Following the amphotericin infusion, we encouraged the patients to move out of bed and engage in light activity such as walking, sitting or standing as soon as the IV infusions for the day were completed. We educated the patients' caregivers to help us implement this practice since most of the patients were semi-conscious and not well oriented. However, this approach was not possible for patients who were unconscious or comatose secondary to cryptococcal meningitis. The most challenging patients were those who were unconscious and had no caregivers. In such cases, we engaged the hospital social workers and physiotherapists to assist with patient ambulation and general care. However, the social workers and physiotherapist are generally limited and not readily available. This method of ambulation also helped to avoid bedsores among the bed-ridden patients.

\section{Treatment of phlebitis} Use of a warm compress

In addition to ambulation, we educated the patient caregivers to use a warm compress. This is a traditional method that involves applying a wet and warm clean cloth to the inflamed sites while applying pressure (compresses). This method was also recently described to be effective and inexpensive in the management of phlebitis [14]. This was the easiest and most convenient method for the caregivers in our setting. We encouraged them to apply the warm compress at least twice daily. However, adherence to this method was limited by poor access to hot water during their inpatient stay. Patient attendants had to buy the hot water or occasionally had it delivered from home.

\section{Topical anti-inflammatory drugs}

Topical anti-inflammatory drugs were applied to manage phlebitis in some patients who did not respond well to ambulation or warm compresses. We mainly used topical diclofenac gel and Emami mentho plus ${ }^{\circ}$ balm, which provided quick relief compared to oral analgesics. 
Almost all the patients used them. However, these drugs were not freely available and were unaffordable for some patients or caregivers.

\section{Oral anti-inflammatory medication}

Oral anti-inflammatory drugs are recommended for symptomatic management of amphotericin infusion reactions including phlebitis [6]. In our setting, we routinely administered paracetamol (acetaminophen) in this population to manage headache, chills, fevers, rigors, and phlebitis, all of which are caused by amphotericin infusions. Non-steroidalanti-inflammatory drugs were not used due to the increased risk of renal toxicity with amphotericin co-administration. However, the effectiveness of paracetamol in the management of phlebitis remains unclear and warrants further investigation. The oral route of administration was also a challenge in unconscious patients. In such cases, we inserted a nasogastric tube for drug administration and feeding.

\section{Oral antibiotics}

There were patients who developed grade 4 phlebitis ("pain at the puncture site with erythema, hardening and a palpable venous cord that is $>1 \mathrm{~cm}$, with purulent discharge" [13]) that were persistent, locally advanced, associated with systemic symptoms such as fever, and did not respond to any of the management methods described above. In such cases, we assumed the presence of an infected thrombus and provided antibiotics for these patients. We primarily used oral antibiotics with Gram-positive coverage such as ampicillin-cloxacillin, amoxicillin-clavulanate, or doxycycline to cover possible staphylococcal infection for 5 days. Only $6 \%$ of the participants with phlebitis and $4 \%$ without phlebitis were given antibiotics during hospitalization. We generally avoided antibiotics unless definite signs of infection were present in accordance with good practice and standards of antimicrobial stewardship.

\section{Limitations to the study}

The data and perspective presented herein are based on our experiences in Kampala, Uganda during a longitudinal, multisite cryptococcal meningitis trial. The research support enabled more resources to be available than in routine care. Additional management strategies may be available. Phlebitis and bacterial infections may be a contribution as to why 1 week of amphotericin and flucytosine has lower mortality than 2 weeks of amphotericin [15]. The investigation of single dose $10 \mathrm{mg} / \mathrm{kg}$ of liposomal amphotericin B may also have a distinct benefit of avoiding phlebitis $[16,17]$.

\section{Conclusion}

We have demonstrated that in this Ugandan cohort of HIV-infected patients with cryptococcal meningitis who were treated with amphotericin B deoxycholate, phlebitis occurred in $18 \%$ of patients. However, the management of phlebitis is very challenging in resource-limited settings. We recommend slow intravenous infusion preferably using standard infusion pumps, with routine post amphotericin saline infusion and rotating the IV access site every 3 days to minimize phlebitis. This should be supplemented by ambulation and use of warm compresses. In severe cases, topical and oral antiinflammatory drugs may be used. Antibiotics are generally indicated only when signs of infection are observed. Patients'/caregiver education is very vital in implementing the management strategies. However, altered mentation, limited availability of essential drugs, and medical supplies remain a significant challenge for high-quality nursing care to implement these management strategies to manage amphotericin-induced phlebitis in routine care in resource-limited settings.

\section{Abbreviations}

AIDS: Acquired immune deficiency syndrome; ASTRO-CM: Adjunctive Sertraline for the Treatment of HIV-associated Cryptococcal Meningitis; CSF: Cerebrospinal fluid; HIV: Human immunodeficiency virus; IV: Intravenous; KCl: Potassium chloride; UNCST: Uganda National Council of Science and Technology; WHO: World Health Organization

\section{Acknowledgements}

We thank institutional support from Drs. Andrew Kambugu and Barbara Castelnuovo. We also thank the entire ASTRO-CM trial team for patients' care.

\section{Authors' contributions}

CA, RK conceived and designed concept. CA, JFN, FK, AS performed data collection. RK, KHH analysed data. CA, RK participated in initial manuscript drafting. CA, RK, JFN, FK, AS, KHH, DAW, JR, DRB, DBM participated in critical revisions for intellectual content. JR, DRB, DBM participated in obtaining funding. DAW, JR, DRB, DBM participated in administrative, technical, or material support. All authors have read and approved the manuscript.

\section{Funding}

This work received no specific funding. However, the parent study (ASTROCM) was supported by the Fogarty International Center and National Institute of Neurologic Disorders and Stroke (R01NS086312, K01TW010268). The funders of ASTRO-CM were not involved in study design, data collection, analysis, interpretation, writing report and the decision to submit the article for publication. DBM and RK are currently supported through the DELTAS Africa Initiative grant \# DEL-15-011 to THRiVE-2, from Wellcome Trust grant \# 107742/Z/15/Z and the UK government.

\section{Availability of data and materials}

The datasets used and/or analysed during the current study are available from the corresponding author on reasonable request.

\section{Ethics approval and consent to participate}

All research participants or their surrogates provided written informed consent. Ethical approval was obtained from the Uganda National Council of Science and Technology (UNCST), Mulago Hospital Research and Ethics Committee, and the University of Minnesota.

Consent for publication Not applicable 


\section{Competing interests}

The authors declare that they have no competing interests.

\section{Author details}

'Infectious Diseases Institute, College of Health Sciences, Makerere University, Kampala, Uganda. ${ }^{2}$ Division of Biostatistics, School of Public Health, University of Minnesota, Minneapolis, USA. ${ }^{3}$ Division of Infectious Diseases and International Medicine, Department of Medicine, University of Minnesota, Minneapolis, MN, USA. ${ }^{4}$ Department of Medicine, School of Medicine, College of Health Sciences, Makerere University, Kampala, Uganda.

Received: 24 April 2019 Accepted: 19 June 2019

Published online: 26 June 2019

\section{References}

1. Durski KN, Kuntz KM, Yasukawa K, Virnig BA, Meya DB, Boulware DR. Costeffective diagnostic checklists for meningitis in resource-limited settings. J Acquir Immune Defic Syndr. 2013;63(3):e101-8.

2. Rajasingham R, Smith RM, Park BJ, Jarvis JN, Govender NP, Chiller TM, Denning DW, Loyse A, Boulware DR. Global burden of disease of HIVassociated cryptococcal meningitis: an updated analysis. Lancet Infect Dis. 2017:17(8):873-81.

3. Parkes-Ratanshi R, Achan B, Kwizera R, Kambugu A, Meya D, Denning D. Cryptococcal disease and the burden of other fungal diseases in Uganda; where are the knowledge gaps and how can we fill them? Mycoses. 2015; 58(S5):85-93.

4. Guidelines for the diagnosis, prevention and management of cryptococcal disease in HIV-infected adults, adolescents and children [http://www.who. int/hiv/pub/guidelines/cryptococcal-disease/en/]

5. Enoch D, Ludlam $H$, Brown N. Invasive fungal infections: a review of epidemiology and management options. J Med Microbiol. 2006;55(7): 809-18.

6. Abassi M, Boulware DR, Rhein J. Cryptococcal meningitis: diagnosis and management update. Curr Trop Med Rep. 2015;2(2):90-9.

7. Ndyetukira JF, Kwizera R, Kugonza F, Ahimbisibwe C, Namuiju C, Sadiq A, Namudde A, Williams D, Abassi M, Rhein J, et al. The conundrum of clinical trials and standard of care in sub-Saharan Africa - the research nurse perspective. J Res Nurs. 2019. https://doi.org/10.1177/1744987118824625.

8. Rhein J, Morawski BM, Hullsiek KH, Nabeta HW, Kiggundu R, Tugume L, Musubire A, Akampurira A, Smith KD, Alhadab A. Efficacy of adjunctive sertraline for the treatment of HIV-associated cryptococcal meningitis: an open-label dose-ranging study. Lancet Infect Dis. 2016; 16(7):809-18.

9. Boulware DR, Rolfes MA, Rajasingham R, von Hohenberg M, Qin Z, Taseera K, Schutz C, Kwizera R, Butler EK, Meintjes G, et al. Multisite validation of cryptococcal antigen lateral flow assay and quantification by laser thermal contrast. Emerg Infect Dis. 2014;20(1):45-53.

10. Dyal J, Akampurira A, Rhein J, Morawski BM, Kiggundu R, Nabeta HW, Musubire AK, Bahr NC, Williams DA, Bicanic T, et al. Reproducibility of CSF quantitative culture methods for estimating rate of clearance in cryptococcal meningitis. Med Mycol. 2016;54(4):361-9.

11. Khoo S, Bond J, Denning DW. Administering amphotericin B-a practical approach. J Antimicrob Chemother. 1994;33(2):203-13.

12. Maki DG, Ringer M. Risk factors for infusion-related phlebitis with small peripheral venous catheters: a randomized controlled trial. Ann Intern Med. 1991;114(10):845-54

13. Urbanetto JS, Peixoto CG, May TA. Incidence of phlebitis associated with the use of peripheral IV catheter and following catheter removal. Rev LatAm Enfermagem. 2016:24:e2746.

14. Annisa F, Nurhaeni N, Wanda D. Warm water compress as an alternative for decreasing the degree of phlebitis. Comprehensive Child Adolesc Nurs. 2017:40(sup1):107-13.

15. Molloy SF, Kanyama C, Heyderman RS, Loyse A, Kouanfack C, Chanda D, Mfinanga S, Temfack E, Lakhi S, Lesikari S, et al. Antifungal combinations for treatment of cryptococcal meningitis in Africa. N Engl J Med. 2018;378(11): 1004-17.

16. Jarvis JN, Leeme TB, Molefi M, Chofle AA, Bidwell G, Tsholo K, Tlhako N, Mawoko N, Patel RKK, Tenforde MW, et al. Short course high-dose liposomal amphotericin B for HIV-associated Cryptococcal meningitis: a phase-II randomized controlled trial. Clin Infect Dis. 2018;68(3):393401.
17. Lawrence DS, Youssouf N, Molloy SF, Alanio A, Alufandika M, Boulware DR, Boyer-Chammard T, Chen T, Dromer F, Hlupeni A, et al. AMBlsome therapy induction OptimisatioN (AMBITION): high dose AmBisome for Cryptococcal meningitis induction therapy in sub-Saharan Africa: study protocol for a phase 3 randomised controlled non-inferiority trial. Trials. 2018;19(1):649.

\section{Publisher's Note}

Springer Nature remains neutral with regard to jurisdictional claims in published maps and institutional affiliations.
Ready to submit your research? Choose BMC and benefit from:

- fast, convenient online submission

- thorough peer review by experienced researchers in your field

- rapid publication on acceptance

- support for research data, including large and complex data types

- gold Open Access which fosters wider collaboration and increased citations

- maximum visibility for your research: over $100 \mathrm{M}$ website views per year

At $\mathrm{BMC}$, research is always in progress.

Learn more biomedcentral.com/submissions 